Conclusion Our ESD pilot has allowed us to develop a COPD service with IT at its heart. This has led to many improvements including a novel daily in-patient virtual MDT wardround.

Please refer to page A192 for declarations of interest related to this abstract.

\section{P75 A COMPARISON OF TELEPHONE VERSUS FACE-TO-FACE CONSULTATIONS WHEN COMMENCING CPAP THERAPY FOR OBSTRUCTIVE SLEEP APNOEA}

MV Foster, MTC Thomas, S Mitchell, T McKown, SE Craig. Liverpool University Hospitals NHS Foundation Trust, Liverpool, UK

\subsection{6/thorax-2021-BTSabstracts. 185}

Background The Covid-19 pandemic has led to the cancellation of a large majority of face-to-face clinic appointments across the NHS, prompting a shift towards telephone reviews. Although perceived as being inferior in some circumstances where direct patient contact is vital for picking up on physical signs and non-verbal cues, for many areas of medicine this could increase efficiency and minimise disruption to patient's lives whilst delivering comparable outcomes. ${ }^{1}$ In this study, we compared CPAP compliance in patients with obstructive sleep apnoea reviewed via telephone with those seen in face-to-face clinics.

Methods Forty patients who attended for CPAP set up were allocated to receive either telephone or face-to-face compliance appointments with twenty patients in each cohort. They were reviewed at 48 hours post CPAP initiation, seven days, one month and six months. At each review their apnoea-hypopnoea index (AHI) and compliance percentage was downloaded. An unpaired T-test was used to compare the two groups.

Results There were 14 males and 6 females in the telephone cohort versus 15 males and 5 females in the face-to-face cohort. The telephone group were aged 32-69 (median age 53) whereas the face-to-face group were aged 30-66 (median age 46).

The mean (SD) compliance at six months in the telephone cohort was $43.8 \%(36.7 \%)$, compared with $45.6 \%(38.5 \%)$ in the group who had had face-to-face reviews $(p=0.869)$. The

Abstract P75 Table 1 The mean (standard deviation) of CPAP compliance and apnoea-hypopnoea index in telephone and face-toface groups at various time points

\begin{tabular}{lllllll}
\hline & Compliance & \multicolumn{5}{c}{ AHI } \\
\hline & Telephone & Face-to-Face & p- \\
value & Telephone & $\begin{array}{l}\text { Face-to- } \\
\text { Face }\end{array}$ & $\begin{array}{l}\text { p- } \\
\text { value }\end{array}$ \\
\hline Initial & n/a & n/a & n/a & $38.6(19.3)$ & $39.5(24.3)$ & n/a \\
48 Hours & $71.5 \%$ & $61.7 \%$ & 0.154 & $3.8(3.6)$ & $6.3(7.8)$ & 0.007 \\
& $(37.0 \%)$ & $(44.6 \%)$ & & & & \\
7 Days & $58.0 \%$ & $57.2 \%$ & 0.840 & $4.7(4.5)$ & $5.1(4.6)$ & 0.661 \\
& $(40.5 \%)$ & $(40.3 \%)$ & & & & \\
1 Month & $54.8 \%$ & $47.5 \%$ & 0.457 & $3.9(3.0)$ & $5.9(4.8)$ & 0.092 \\
& $(36.2 \%)$ & $(38.4 \%)$ & & & & \\
6 & $43.8 \%$ & $45.6 \%$ & 0.869 & $3.5(2.8)$ & $5.6(4.5)$ & 0.057 \\
Months & $(36.7 \%)$ & $(38.5 \%)$ & & & & \\
\hline
\end{tabular}

mean (SD) AHI at six months in the telephone cohort was 3.5 (2.8), compared with 5.6 (4.5) in the face-to-face group $(\mathrm{p}=0.057)$. The results show no significant difference between the two groups.

Conclusion Conducting telephone consultations with patients who are being set up on CPAP was shown to be non-inferior to having face-to-face reviews. This study supports the idea that telephone reviews are a safe alternative to traditional face-to-face clinics.

\section{REFERENCE}

1. Car J, Koh GCH, Foong PS, Wang CJ. Video consultations in primary and specialist care during the covid-19 pandemic and beyond. BMJ 2020;371:m3945. doi: https://doi.org/10.1136/bmj.m3945

\section{P76 IMPROVING COMMUNITY-BASED CARE USING ONLINE COMMUNICATION PORTAL FOR PATIENTS WITH AN INDWELLING PLEURAL CATHETER}

J Liang, K Ur Rehman, P Sivakumar. Guys and St Thomas' NHS Foundation Trust, London, UK

\subsection{6/thorax-2021-BTSabstracts. 186}

Introduction and Objectives Indwelling pleural catheter (IPC) manufacturers offer an online portal to facilitate communication between secondary care services and the district nurses (DNs).

We aimed to characterise the experiences of patients and DNs of community-based IPC care that is supported by an online communication portal.

Methods A manufacturer-based online portal was adopted by our service in February 2021 with data prospectively collected on IPC care. Qualitative feedback was sought from patients and district nursing teams during follow-up.

Results 13 eligible patients underwent IPC insertion between February 2021 and June 2021. 100\% (13/13) patients agreed to be enrolled onto the portal.

Seven issues affected six patients during follow up, four of which were addressed in the community.

Four phone calls were documented regarding bottle shortages. Via the portal, overnight delivery of bottles to patient's address was organised on all occasions. DNs were also trained on fast-track bottle requisition.

In three patients, sutures were not removed as instructed, which was identified at planned hospital follow up. Via the portal, the manufacturer contacted DNs to organise refresher training. Two DN teams accessed this course and reported benefits, finding it particularly useful for their novice practitioners. The training covered all aspects of the product, drainage procedure, ordering process and troubleshooting.

All patients reported satisfaction with their communitybased care during the follow up period. The four patients with bottle issues were grateful for a prompt resolution without the need to attend the hospital.

One patient reported a 'sense of confidence in the care being provided in the community.'

Conclusion The online portal ensures seamless communication between a secondary care pleural service and the local district nursing teams, avoiding the need for healthcare attendance through prompt resolution of community-based issues. It also facilitates the provision of community-based training. The partnership between these teams ensures better care quality, clinical outcome and support for patients. 